\title{
Third International Symposium on the Neuronal Ceroid-Lipofuscinoses (Batten's Disease), Indianapolis, Indiana, USA
}

A symposium on the neuronal ceroid-lipofuscinoses (NCL), of which Batten's disease is the prototype disorder, was held in Indianapolis from 30 May to 1 June 1990. This symposium, organised by Drs Aristotle Siakotos and Glyn Dawson, consisted of five sessions; of these, one session was devoted to the mapping of NCL genes, an area in which dramatic advances have been made recently.

The symposium was composed of specialists in neurology, neuropathology, paediatrics, molecular genetics, biochemistry, and veterinary medicine. A unique feature of this conference was the attendance of parents of NCL children. These families provided many of the blood cell DNA samples used in the locational (positional) cloning studies.

The NCL, as a group of inherited disorders, have an incidence estimated to be as high as 1/12 500 live births, with approximately 440000 carriers in the USA. These neurogenetic disorders are a frequent source of progressive seizures in children and are now considered to be the major storage diseases in which the biochemical defect remains unknown.

In the first session, Krystyna Wisniewski discussed neuropathological diagnoses, classification, and heterogeneity in the NCL. Among the 221 cases studied, heterogeneity (pathological and clinical) was estimated to be 10 and $20 \%$, respectively. Rose-Marie Boustany reported on the geographical distribution and ethnic background of persons with NCL. The majority of well diagnosed cases were traced back to northern Europe; however, several other participants pointed out that many Batten patients were now being discovered in areas not previously surveyed, including the Near and Far East. This unexpected Batten distribution may be a consequence of the difficulty in diagnosing these disorders, usually after all other lysosomal storage disorders are eliminated from the diagnosis. Sophisticated biochemical procedures to screen suspected patients are generally not available in Third World areas.

The session on mapping of NCL genes was chaired by Dr Michael Conneally. The genetic defect(s) have not yet been described, despite years of research on the basic biochemistry of these disorders. An alter- native approach is to use the strategy of locational (positional) cloning. This approach attempts to find the approximate location of the defective gene(s) in the genome through linkage analysis, and then use molecular genetic techniques to identify the exact genetic defect(s). The application of this approach to NCL has resulted in advances in two of the known forms of NCL.

Restriction fragment length polymorphism (RFLP) studies have led to the conclusion that one gene for the most common (juvenile) form of NCL (CLN3) was linked to the haptoglobin locus on chromosome 16q (Eiberg et al, 1989). Dr Gardiner presented an update of this work with linkage data on 23 informative families from Europe. Many anonymous RFLPs were tested in this region, and a peak location score of over 10 has now been found. The most likely location of the CLN3 gene is near the D16S148 locus (only one recombinant has been observed so far). No evidence for locus heterogeneity was seen among these families.

In a separate sample of eight CLN3 families from North America, Dr Haines reported positive, but not significant, lod scores in this region. His analysis suggested the possibility of heterogeneity, as one family with confirmed CLN3 had two recombinant events with D16S148. Both researchers agreed that more family studies were necessary, as is the identification of other informative probes in this region.

The gene for another form of NCL, the Finnish infantile type (CLN1), was localised to the short arm of chromosome 1 in a report by Peltonen et al. A lod score of $4.0(\theta=0)$ at DIS79 was reported, and a tentative localisation between DIS57 and DIS79 was suggested. There was no evidence of heterogeneity among these families.

The gene for the late infantile form of NCL (CLN2) has not been localised. Although CLN2 could be allelic either to CLN3 or CLN1, a third locus cannot be ruled out. Obtaining enough families for study has been the primary difficulty, and all researchers agreed that efforts in this area must be increased.

New research developments included assessments of the role of phospholipase abnormalities and 
derangements in lipid metabolism. Dawson reported a phospholipase $A_{1}$ deficiency in canine NCL brain. Siakotos and van Kuijk found that 4-hydroxynonenal levels in stimulated platelets and neutrophils were promising as markers of the affected and carrier state in canine NCL. Ivy found that the intraventricular injection of selected protease inhibitors (leupeptin, $\mathrm{E} 64 \mathrm{C}$ ) in the rat induced the accumulation of lipopigments similar to the disease specific lipopigments stored in the NCL English setter. She proposed NCL may be a result of a defect in protein metabolism. The analysis of stored biochemical substrates, which has been successful in other lysosomal disorders, has yielded only evidence of protein and dolichol derivatives. Among these stored proteins, Palmer and Jolly have reported very high concentrations of the c subunit of ATP synthase in sheep affected with NCL, and in both the juvenile and late infantile forms of human NCL. The $8 \mathrm{kd}$ hydrophobic protein constituted over $50 \%$ of the isolated storage bodies. In contrast, Katz and Gerhardt (1990) have suggested that methylation of proteins could generate the pathognomonic autofluorescent pigment. Wisnieski et al reported the storage of amyloid $\beta$ protein precursor in neurones and lipopigment granules of late infantile, juvenile, and adult forms of NCL, as well as Alzheimer's neural tissue. NCL may be the result of decreased or defective protein catabolism, but the mechanism which results in the build up of either of these proteins has not been elucidated.

In all forms of NCL the storage bodies show massive accumulation of dolichol pyrophosphoryl oligosaccharides. Hall concluded that this phenomenon may be a result of irreversible storage rather than abnormal synthesis. In contrast, Daniels suggested that dolichol phosphate sugar metabolism may be defective in NCL.

A section on animal models of NCL included similarities and differences between the various model systems and the human disorders. Briefly, nearly all of the canine models approximate to the human juvenile disease (CNL3), but the Tibetan terrier was shown by Riis to be very similar to the human adult onset condition (Kufs' disease).

The final session on new approaches to therapy focused on allogenic bone marrow transplantation (BMT) in lysosomal storage disorders. Taylor reported BMT used by an Australian team as highly effective in preventing the onset of symptoms in fucosidosis in the English Springer spaniel. Animals receiving BMT at 2 to $\mathbf{4}$ months remained symptom free over five years later, well beyond the maximum four year life span of affected animals, but transplants performed on older animals were ineffective. Krivit presented data on seven human metachromatic leucodystrophy patients with BMT. Intervention with BMT in the very early stage of the disease led to marked reductions in the severity of the disease.

The final session of the conference was devoted to families of Batten's disease patients with a question and answer period, followed by a request for more family participation in the continuing linkage analysis studies. Over 80 blood samples were collected, and the follow up total has exceeded 100. All of these families are also included in the NCL bank of immortalised lymphocytes under the supervision of Dr Jay Tischfield of the Department of Medical Genetics at the Indiana University School of Medicine. This facility is supported by the Children's Brain Disease Foundation.

Unlike other lysosomal storage disorders many of the stored materials associated with NCL are probably secondary to the as yet unknown primary defect: included are the dolichols, dolichol pyrophosphoryl oligosaccharides, the c subunit of ATP synthase, and various other lipopigment constituents. A striking feature of NCL research is the availability of many animal models of NCL. In the dog many of these models are similar; however, the Tibetan terrier analogue of Kufs' disease is a major resource, since this disorder is extremely rare in the human population. The final session on the application of BMT to reduce or eliminate the symptoms of fucosidosis in dogs with this disorder was very striking. The studies of Krivit on human metachromatic leucodystrophy patients indicates that, even in this disorder, very early intervention with BMT in presymptomatic persons can result in correction of the metabolic disturbance and amelioration of the disease. However, success of BMT depends on the availability of a specific biochemical procedure for detecting presymptomatic but affected persons. In NCL this requirement remains equivocal. If progress in NCL research continues at the present accelerating pace, this goal may soon be achieved in this group of disorders, which were first described in Roros, Norway in 1826.

ARISTOTLE SIAKOTOS

Department of Pathology, Indiana University

School of Medicine, Indianapolis, IN 46202, USA.

JONATHAN HAINES Molecular Neurogenetics Laboratory, Massachusetts General Hospital, Charlestown, MA 02129, USA.

GLYN DAWSON

Department of Pediatrics, University of Chicago School of Medicine, Chicago, IL 60637, USA. 